\title{
ROBERT DESCIMON, LES SEIZE ET LA SAINTE LIGUE
}

D'une érudition presque classique, l'étude que $\mathbf{R}$. Descimon a consacrée aux "Seize " se révèle, en outre, une importante contribution au savoir scientifique. En effet, elle participe au renouvellement conceptuel qu'exige aujourd'hui l'histoire tout en approfondissant notablement nos connaissances sur la Ligue ${ }^{1}$. Cet approfondissement et ce renouvellement sont d'ailleurs indissociables, car ils s'accomplissent l'un par l'autre et n'existeraient donc pas l'un sans l'autre. Cette liaison exige donc au départ de cet essai, une évocation rapide de l'historiographie ligueuse, ou plus exactement de ce qu'il est nécessaire d'en exposer pour comprendre et jauger la démarche et les apports de l'auteur.

Longtemps, la Ligue passa pour un déchaînement furieux de masses populaires fanatisées. Sans doute forgée par la propagande d'un roi victorieux, cette image simpliste remontait aux lendemains memes des troubles. Elle possédait un immense avantage, d'ailleurs ambigu, qui lui assura une longue survie. En effet, aux yeux de ceux qui croyaient détenir le " vrai savoir " du moment, elle justifiait la nécessité d'encadrer un peuple incapable de " penser juste " et d'" agir raisonnablement " pour le " libérer » des opiums obscurantistes et contenir sa sauvagerie naturelle. Préparé dès 1937 par le livre pionnier de $\mathrm{H}$. Drouot ${ }^{2}$, mais véritablement lancé dans les anncés soixante-dix sous l'impulsion de J. H. M. Salmon et de D. Richet ${ }^{3}$, le renouveau actuel des études sur la Ligue est en fait la négation de cette vision traditionnelle. Né d'une critique plus aiguë des narrations contemporaines et d'un recours à un éventail documentaire plus élargi, il est conscience des insuffisances de l'interprétation classique et davantage encore des distorsions

1. Robert Descimon, « Qui étaient les Seize ? Mythes et réalites de la Ligue parisienne (1585-1594) ", tiré à part des Mémoires de la Fédération des sociétes historiques et archéologiques de Paris et Ile-de-France, t. XXXIV, Paris, Klincksieck, 1983, p. 7-300.

2. Henri Drouot, Mayenne et la Bourgogne, Dijon, Imprimeurs Bernigaut et Privat, 1937.

3. Par leurs séminaires comme par les travaux qu'ils ont guidés et dirigés.

Revue de synthèse : IV $\mathrm{S}^{\mathrm{N}} \mathrm{N}^{\circ} 2$, avril-juin 1987. 
introduites par les liens qu'elle entretint, dès l'origine, avec les événements eux-mêmes. Il est découverte d'une présentation orthodoxe nécessaire, mise en place pour conjurer les miasmes du mouvement et escamoter la compromission de serviteurs dévoyés, rentrés dans le "droit chemin " et métamorphosés en zélés propagandistes et thuriféraires convaincus. Cette simple prise de position conduit à insister encore plus sur l'aspect social de l'insurrection tout en le recentrant plus haut dans la hiérarchie et en l'assaisonnant de politique; elle relègue, en revanche, au second plan ce qui relève du religieux et du culturel pourtant implicitement au cour de l'ancienne représentation.

Or cette signification sociale de la Ligue, désormais mise en avant, se révéla ambiguë au fur et à mesure que se multipliaient les analyses urbaines ponctuelles (en particulier sur Amiens, Angers, Dijon, Nantes, Rennes, Rouen, sans oublier Paris) 4. Les uns découvrirent les revendications d'une couche moyenne (essentiellement la basoche ou les artisans) bloquće dans son ascension par les corps qui la dominaient (avant tout la robe et la marchandise). Les autres, au contraire, rencontrèrent des réseaux de clientèle rivaux fracturant du haut en bas l'ensemble de la société. Devant ces résultats aussi contradictoires que solidement argumentés, l'idée s'insinua, sous l'influence de "l'empirisme anglo-saxon " ', qu'il fallait substituer à un soulèvement monolithique au-delà de ses multiples aspects, au moins deux types de conflits sans grands liens entre eux, exacerbés à la faveur d'un affaiblissement du pouvoir central et puisant dans le refus d'un roi protestant l'illusion d'un combat commun, illusion qu'ils transmirent aux historiens " piégés » par les mots.

Conscient et inquiet de cet " éparpillement du sens " 6, Robert Descimon a choisi de se placer au coeur du mouvement dans son expression la plus radicale, afin de déterminer s'il convenait ou non de troquer l'" unité " pour la " pluralité ». En conséquence, il s'est livré à une analyse prosopographique de deux cent vingt-cinq meneurs parisiens (les « Seize » au sens large) qu'il repéra à l'aide d'un jeu subtil de douze critères, cinq majeurs " tenus pour suffisants en eux-mêmes » et sept mineurs « simples indices dont la conjonction forme des présomptions

4. Élie Barnavi, Le Parti de Dieu. Étude sociale et politique des chefs de la Ligue parisienne, 1585-1594, Louvain, Nauwelaerts, 1980 ; Philip BENEDICT, Rouen during the Wars of Religion, Cambridge, Cambridge U.P., 1981 ; Robert Harding, " Revolution and Reform in the Holy League : Angers, Rennes, Nantes ", Journal of Modern History, 1981, p. 379-416 ; Arlette Lebigre, La Révolution des curés, Paris, 1588-1594, Paris, Albin Michel, 1980 ; Denis RiCHET, « Aspects socio-culturels des conflits religieux à Paris dans la seconde moitié du xvie siècle ", Annales E.S.C., 1977, 4, p. 764789 ; David Rosenberg, Social Experience and Religious Choice. A Case Study, the Protestant Weavers and Woolcombers of Amiens in the Sixteenth Century, unpublished Ph. D. Dissertation, Yale University, 1978 ; John H. M. SAlmon, " The Paris Sixteen 1584-1594 : The Social Analysis of a Revolutionary Movement ", Journal of Modern History, 1972, p. 540-576.

5. R. Descimon, op. cit. supra n. 1, p. 25.

6. Ibid. 
convaincantes $\gg 7$. Il a toutefois exclu de son corpus les ecclésiastiques parce que " le magistère clérical s'exerce dans sa sphère propre " et que " l'Église poursuivait dans la Ligue ses propres fins ", même si le « radicalisme bourgeois " en " reprenait " une " partie à son compte " 8 .

Au terme de cette analyse prosopographique, dont la place au cour même de l'ouvrage souligne le rôle décisif qu'y attache l'auteur, la conclusion de R. Descimon surprend au premier abord : il ne choisit pas entre une insurrection unique et la convergence chronologique de mouvements différents ; en d'autres termes, il renonce à trancher entre deux positions apparemment exclusives l'une de l'autre. Mieux, il prétend que l'" unité " se retrouve dans la " pluralité ", une " pluralité " qu'il affirme même plus complexe et plus variée que ne l'estimaient ses prédécesseurs et son amical rival Élie Barnavie 9. Il déclare en effet, que

"l'on doit [en premier lieu] reconnaître que la prise de parti ligueuse se nourrissait de mobiles différents selon les classes : clientélisme intéressé des oligarques, lutte de corps qui mobilisait une partie de la basoche contre la robe, effort tragique des marchands pour conserver fortune et notabilité... »

"Cette incontournable diversité des adhésions " résulterait de clivages d'ordre politique (acceptation ou refus des « violences collectives $")$, économique (" dynamisme " ou " atonie " des différents secteurs), social (mobilité des uns et stagnation des autres) ou familial (isolement ou incorporation à des parentèles plus ou moins étendues) ${ }^{10}$. Néanmoins, cette diversité n'empêcherait nullement des comportements et des actions similaires.

Dès lors, le problème est de reconnaître si cette confluence de motivations variées dans des attitudes semblables traduit une simple rencontre fortuite favorisée par l'imprégnation religieuse contemporaine ou si, au contraire, elle signifie que

7. Ibid., p. 77-84. Les cinq critères majeurs sont : « Avoir participé à la fondation de la Seconde Ligue et à ses premiers développements " - " Avoir appartenu aux différents conseils de la Ligue durant les années 1589-1591 " - " Avoir eté investi par le parti d'une charge, d'une autorite ou d'une mission of ficielles " - " Avoir engage son credit personnel dans l'effort financier consenti par les Seize lors des sièges de Paris 》 - " Avoir eu une responsabilité politique ou une part d'initiative dans la préparation et l'exécution du meurtre du président Brisson » et des conseillers Larcher et Tardif. Les sept critères mineurs sont : "Avoir exercé une charge bourgeoise impliquant représentation de la communauté parisienne " - "Avoir eté victime des proscriptions bourboniennes au printemps 1594 " - "Etre mentionné comme membre des Seize ou Ligueur avancé dans les mémoires du temps " "Avoir eu une part active dans des démarches, des affaires de police, etc., d'esprit extrémiste et partisan, sur commission du conseil général de l'Union ou de l'Hótel de Ville ou bien de son initiative privée " - " Avoir proféré des injures partisanes " - " Pour ceux qui occupaient à Paris une place au sein de la notabilité, avoir servi dans l'armée de l'Union hors des murs de Paris, soit en qualité d'officier, soit à la suite et dans la garde des princes catholiques " - "Avoir participé à certaines assemblées de ville ou à certaines manifestations d'esprit ligueur avancé ").

8. Ibid., p. 49-50.

9. R. Descimon, "La Ligue a Paris (1585-1594): une révision ", E. Barnavi, " Réponse à Robert Descimon », R. DESCIMON, « La Ligue : des divergences fondamentales ", Annales E.S.C., I, 1982, p. 72-127.

10. R. DESCIMON, op. cit. supra n. 1, p. 296. 
« l'incontournable diversité » n'épuise pas le phénomène ligueur, au moins dans la capitale. A priori, l'effritement de la Ligue parisienne sous le choc d'événements qui, comme l'exécution du président Brisson ", démasquent les mobiles contradictoires de prises de position identiques en apparence, porterait plutôt à pencher en faveur de la première interprétation. L'évolution même de la révolte semblerait, d'ailleurs, le confirmer. Elle s'insère, en effet, dans le modèle qui, selon É. Barnavi, régirait les révolutions européennes, des Hussites du XVe siècle aux Puritains anglais du XVII siècle ${ }^{12}$. En bref, il n'y aurait jamais eu d'union véritable, mais une illusion d'union, une coalition se croyant à tort une union, partant vouée à la décomposition sous le poids de ses propres contradictions. Que ces contradictions soient multiples à Paris et différentes d'une ville à l'autre ne changerait rien au phénomène.

R. Descimon refuse cette hypothèse et s'emploie à la réfuter. Pour ce faire, il développe tour à tour son argumentation sur le plan théorique et sur le plan concret. Sur le plan théorique, il fait remarquer que le modèle d'É. Barnavi n'a pas de valeur probatoire parce que « descriptif, non théorique ", " il fonctionne comme une paraphrase de l'événement $"$. Ce faisant, et sans toutefois le dire explicitement, $\mathbf{R}$. Descimon conteste qu'une identité ou une parenté formelles entre deux ou plusieurs séquences événementielles suffisent pour conclure à une identité ou à une parenté des phénomènes historiques qu'elles expriment. En d'autres termes, se plaçant à contre-courant d'une tendance dominante, il nie, au nom d'une exigence de plus grande " scientificité ", qu'il soit licite d'inférer automatiquement un signifié unique de signifiants analogues. De cette manière, il contribue, sans trop avoir l'air d'y toucher, aux renouvellements conceptuels en cours. En effet, cette prise de position suggère d'abord que le sens d'une séquence événementielle n'est pas contenu uniquement en elle (puisque des séquences semblables peuvent en avoir de différents), mais dans les rapports qu'elle entretient avec d'autres séquences (étant donné qu'eux seuls désormais permettent d'éclairer les séquences et de dépasser le " formel »). Cette attitude laisse, en outre, entendre que le sens des evénements est « ouvert » car, au fur et à mesure de la découverte de nouveaux rapports, il se modifie et se précise.

Sur le plan concret, $\mathbf{R}$. Descimon estime que dans la Ligue la préoccupation religieuse est beaucoup plus qu'un langage commun procurant une apparence d'unité à des conflits fondamentalement dissemblables, parfois inconciliables, à coup sûr étrangers les uns aux autres. Pour lui, elle est le catalyseur qui engendre une réelle unité à partir de "l'incontournable diversité », de la " pluralité » des engagements. " La synthèse, écrit-il, se réalisait dans et par la religion ", expression forte qu'il complète en ajoutant : " on touche à la définition même de l'Union " 13 . Néanmoins, bien que retrouvant ainsi son autonomie, le religieux dans cette optique demeure uniquement un moyen. Et, effectivement, il ne peut en être autrement puisque la fracture religieuse, bien loin de coïncider

11. E. BARnAVi, R. Descimon, La Sainte Ligue, le juge et la potence : l'assassinat du président Brisson, 15 nov. 1591, préf. Denis Richet, Paris, Hachette, 1985.

12. E. Barnavi, Conférence au Collège de France, mai 1983.

13. R. Descimon, op. cit. supra n. 1, p. 296. 
avec la coupure activiste, se situe nettement au-delà de celle-ci. En effet, nombre de « Politiques ", adversaires des Seize, partagent avec ces derniers des " conceptions religieuses qui souvent pourraient se réclamer également de la Contre-Réforme » ; « on pense aux Séguier ou au président Fauchet » 14. Dans ces conditions, si le radicalisme religieux ne constitue que l'agent de la synthèse, l'« unité ligueuse " possède forcément une autre signification plus décisive. En conséquence, pour R. Descimon, « La Ligue est l'expression synthétique d'une sociabilité urbaine revigorée par les guerres civiles ». D'après lui,

" le matériau préexistait, mais son agencement fut inédit. Les diverses consciences de corps débouchaient, à travers la conception de la ville comme corps commun qui représente et synthétise les corps dont elle est composée, sur une sorte de conscience bourgeoise intégrée que fondait le privilège urbain inégal dans son identité »15.

Que cette « conscience bourgeoise » ait dû emprunter la voie insurrectionnelle pour se manifester suppose que, depuis le XVe siècle, elle s'était profondément dégradée sous l'action de puissants dissolvants dont le moindre pourrait bien ne pas avoir été la Robe, et en particulier la Robe Longue. À la fin du XVIe siècle, les officiers auraient été ainsi déchirés entre un idéal citadin déclinant et leur appartenance à un ordre monarchique qui veut s'affirmer ; d'où leur division lors de la crise ; d'où aussi, après le triomphe royal, le désir des plus compromis d'occulter leur " défaillance ", c'est-à-dire leur adhésion passagère à une vision sociale vaincue. Ce faisant, l'interprétation de $\mathbf{R}$. Descimon rejoint et illustre la thèse de Bernard Chevalier sur l'évolution des « bonnes villes de France " ${ }^{16}$, en toute connaissance de cause d'ailleurs, puisque à plusieurs reprises, il s'y réfère explicitement.

En réalité, l'apport de $\mathbf{R}$. Descimon, aboutissement des travaux et des réflexions de tous ceux qui, depuis $H$. Drouot, ont contribué au renouvellement de l'historiographie ligueuse, se situe moins dans les éléments qu'il introduit ou réintroduit (pluralité des mobiles, catalyseur religieux, solidarité urbaine) que dans l'agencement de ces éléments entre eux, c'est-à-dire dans la structure retrouvée, mieux, découverte, d'un phénomène dont il restaure ainsi l'unité. Il suffit, pour s'en convaincre, de comparer son essai à un ouvrage " classique " (au sens un peu désuet du terme) de la production " positiviste » du début du siècle. Dans le cadre de l'Histoire de France des origines à la Révolution dirigée par E. Lavisse, J.-H. Mariéjol a rédigé les chapitres qui intéressent la Ligue ${ }^{17}$. À travers un récit minutieux, il adhère de toute sa volonté à l'interprétation traditionnelle du mouvement. En conséquence, il n'est pas surprenant de l'entendre louer « les conseillers de Mayenne » qui « s'efforçaient de maintenir les formes monarchiques, le gouvernement monarchique, les traditions monarchiques », « tandis que les Seize ne rêvaient que bouleversement, terreur et massacres " ${ }^{18}$. Cepen-

14. Ibid., p. 295.

15. Ibid., p. 296.

16. Bernard Chevalier, Les Bonnes Villes de France, du xIV'au xvi siècle, Paris, Aubier-Montaigne, 1982.

17. Jean-H. MARIÉJOL, La Réforme, la Ligue, l'Édit de Nantes, Paris, Hachette, 1904. 18. Ibid., p. 376. 
dant, cette orthodoxie de l'interprétation s'accompagne d'une attention scrupuleuse aux événements et, çà et la, d'involontaires élans de sympathie vis-à-vis des insurgés ${ }^{19}$. Il en résulte que, soit par indulgente compréhension (un peu honteuse, il est vrai), soit par fidélité aux méthodes scientifiques de l'école " historiciste " française, soit en raison des deux à la fois, J.-H. Mariéjol a bel et bien aperçu les multiples facettes du radicalisme ligueur. Il a su repérer avec un égal bonheur la bigarrure des motivations et ce qu'il nomme un peu maladroitement, mais preuves à l'appui, le « réveil de l'esprit communal » 20 . Traitant de l'« esprit révolutionnaire des Seize ", il signale que " plus d'un d'entre eux aspirait, dans l'intérêt de son parti ou dans le sien, à siéger à la Grand'Chambre ", que " la petite bourgeoisie, qui fournissait les eléments ardents de la Ligue, voulait supplanter l'aristocratie parlementaire et s'asseoir "sur les fleurs de lys" ". Il conclut ainsi : " Les ambitions les plus basses comme les plus hautes, l'esprit de vengeance et l'esprit de parti, l'intérêt de Paris et le sentiment très vif de sa déchéance, tout s'unissait pour aigrir les Seize " ${ }^{21}$. Le mot " aigrir » mis à part, les jugements de valeur mis de côté, on peut douter que R. Descimon ait beaucoup à redire aux affirmations de J.-H. Mariéjol.

Cependant, il ne faudrait pas déduire de ce parallélisme que le travail de R. Descimon se contente de retrouver, au mieux de confirmer, les conclusions de J.-H. Mariéjol et qu'en conséquence le vaste effort de recherches entrepris récemment n'aboutit qu'à une connaissance plus complète des ligueurs provinciaux et des meneurs parisiens dans la grande tradition érudite du XIX' siècle. Que des éléments de l'interprétation soient communs à quelques nuances près ne fait aucun doute. Néanmoins, fidèle aux canons de l'historicisme en vigueur à son époque, J.-H. Mariéjol vise uniquement à établir ces éléments (mobiles divers, militantisme religieux, solidarité urbaine) ; il se borne ainsi à constater leur présence dans le radicalisme ligueur. Pour lui, un phénomène historique se definit donc par ses composants; il n'est que juxtaposition de composants.

$\mathbf{R}$. Descimon pense manifestement autrement. Dans son optique, le mouvement des Seize n'est pas mobiles divers, militantisme religieux, solidarité urbaine ; il n'est même pas tout cela en même temps ; il est relations entre ces mobiles, ce militantisme et cette solidarité. Le phénomène historique devient ici agencement original d'éléments. Cette différence de conception du phénomène historique, en déplaçant l'accent des composants aux rapports qu'ils entretiennent, débouche fatalement sur une autre interprétation du mouvement ligueur, plus attentive à sa structure que celle de J.-H. Mariéjol qui, par principe, n'accordait à celle-ci qu'une attention restreinte, si même il lui en accordait. Dans ces conditions, les deux interprétations ne peuvent pas être identiques, parce qu'elles relèvent de deux vues dissemblables du phénomène historique.

Quelles sont donc les relations fondamentales qui, selon R. Descimon, unissent les mobiles particuliers, le militantisme religieux et la solidarité urbaine,

19. Ibid., Livre IV, passim, en part., p. 418-419.

20. Ibid., p. 377.

21. Ibid., p. 375. 
et définissent ainsi l'activisme ligueur ? Elles seraient essentiellement d'intégration, intégration des mobiles particuliers à la solidarité urbaine sous l'action du militantisme religieux, d'où « l'incontournable diversité des adhésions vécues à l'idéal commun d'une cité épurée ", " synthèse [réalisée] dans et par la religion $" 22$. À notre avis, le rapprochement de ces deux expressions voisines, mais non contiguës, livre la clé de l'interprétation globale de l'auteur contenue dans un discours qui, se moulant directement sur la progression de l'enquête et la démarche de la pensée, exprime ses résultats avec prudence, laissant le lecteur en tirer les conclusions systématiques qu'il suggère. Mais si celles-ci sont telles qu'elles viennent d'être formulées, la contribution de $\mathbf{R}$. Descimon aux efforts de réflexion conceptuelle contemporains est loin d'être négligeable parce que, s'appuyant sur un cas concret, elle permet tout à la fois d'en éprouver l'efficacité et d'en affiner les enseignements.

Par sa définition de la Ligue radicale, l'auteur se distingue, en effet, non seulement d'un positiviste comme J.-H. Mariéjol, mais aussi de ses prédécesseurs immédiats. Pour ceux-ci, il s'agissait encore d'expliquer le phénomène ligueur extrémiste en en établissant la cause principale et en en reconnaissant ainsi la " nature " (sociale ou politique ou religieuse, etc.). Ce faisant, ils transposaient en histoire une pratique philosophique ancienne qui se fixait pour buts la recherche de la cause première et la détermination de l'essence des choses. Comme les causes premières ou les essences des choses sont, pour un même phénomène, exclusives les unes des autres, il était fatal que si plusieurs paraissaient également fondées, il en résultât des débats sans fin propres à alimenter d'inépuisables querelles, ou une attitude empirique, sinon sceptique, qui finissait par détruire l'unité du phénomène. En considérant un phénomène historique (ici le radicalisme ligueur) comme une coalescence et un agencement d'éléments, R. Descimon s'inscrit dans une orientation de pensée qui tend à sortir l'histoire d'une impasse. Car, de cette manière, il devient possible de tenir compte en même temps d'observations raisonnablement assises qui, auparavant, apparaissaient pourtant contradictoires, parce qu'on les considérait comme des « causes principales » ou des « qualités » révélatrices d'une " nature ».

Une telle attitude ne signifie pas pour autant que l'historien, en dégageani des systèmes relationnels, renonce à ce qui est sa raison d'etre : saisir des évolutions. Il continue à les envisager, mais à travers la formation, les transformations et la désintégration de ces systèmes. Revenons à la Ligue et risquons une hypothèse (dont bien des éléments se retrouvent d'ailleurs chez $\mathbf{R}$. Descimon et ses prédécesseurs). Pour autant qu'on le sache, la période ligueuse se situe au terme d'une désagrégation économique et politique commencée environ un quart de siècle auparavant. La renaissance progressive des difficultés économiques, l'affaiblissement tendanciel d'un pouvoir royal miné par les factions et incapable de choisir entre les réformes religieuses constituent autant de nouveautés auxquelles les citadins doivent désormais faire place dans leurs représentations du monde dans lequel ils vivent. Or les images alors courantes basées sur une certaine prospérité, une

22. R. Descimon, op. cit. supra n. 1, p. 296. 
puissance princière en voie d'affirmation et l'unité du christianisme occidental, se révèlent, de ce fait, inaptes à cette tâche. En conséquence, il se pourrait que se développe ainsi dans le royaume une crise des représentations semblable à celle décrite par Ph. Mack Crew pour les Pays-Bas vers 1565-1566 23. Certes, dans ces mêmes années soixante, certaines villes comme La Rochelle, Montauban ou Nîmes restructurent leur vision du monde autour d'un protestantisme qui leur fournit une explication de leurs heurs et malheurs. Toutefois, en rejetant cette vision, parfois violemment, les autres cités ont du mal à intégrer dans un système cohérent la désagrégation qui se poursuit sous leurs yeux malgré les efforts tentés pour l'enrayer, et s'accélère même au cours de la décennie quatre-vingt pour culminer, après 1584 , avec la perspective d'un roi huguenot.

Le recours apparaît néanmoins peu à peu. Les habitants des villes se replient sur la vieille solidarité citadine d'autrefois, menacée mais non disparue, d'autant que cette solidarité s'accroche à d'autres solidarités, religieuses cette fois, dont elle ne se distingue pas toujours nettement parce qu'elles s'ancrent toutes dans des institutions et des pratiques voisines (comme les confréries et les métiers), corrélées à défaut d'être identiques ; d'où l'adhésion à un idéal commun, celui des bonnes villes. Mais cet idéal devient également « porteur d'espoir ». Dans sa réalisation, chaque individu, chaque groupe, voit maintenant l'accomplissement de ses propres aspirations. Celles-ci s'incorporent de cette manière, " dans et par la religion " dans la vision d'un ordre citadin à rénover, ce qui, selon R. Descimon, définit, nous l'avons vu, l'activisme ligueur. Cependant, l'action des Seize acquiert de ce fait une ambivalence, puisqu'elle manifeste tout à la fois des mobiles particuliers et des motivations générales. Dès lors, le contenu des mobiles particuliers étant variables et celui des motivations générales, unique, les relations d'intégration qui fondent l'Union sont à tout moment menacées de se désagréger en raison des antagonismes dont, ensemble, elles sont porteuses. Par leurs interventions, Henri IV, voire Mayenne, n'ont fait que précipiter une évolution vraisemblablement fatidique. La Sainte Ligue, dans sa version des Seize, serait en conséquence un système relationnel apparu progressivement pour pallier la décomposition d'autres systèmes, mais ses contradictions internes dues aux éléments qu'il rassemble et l'interférence d'un système monarchique encore bien vivant quoique ébranlé, l'auraient acheminé vers sa désintégration et sa ruine.

R. Descimon affirme que " les Seize n'étaient pas ce qu'on vous a dit 》24. Avec lui, comme avec d'autres, grâce à un effort thérique s'enracinant dans une recherche concrète, sans par ailleurs rien renier de son passé (ce serait un comble) mais en s'appuyant sur celui-ci pour progresser, l'histoire n'est plus tout à fait ce que l'on croyait et imaginait.

Hugues NeveuX, Université de Paris $X$ - Nanterre.

23. Phyllis MaCk Crew, Calvinist Preaching and Iconoclasm in the Netherlands, 1544-1569, Cambridge, Cambridge U.P., 1978, en part., chap. VI, p. 140-181.

24. R. DESCIMON, op. cit. supra n. 1, p. 295. 